Our case must be distinguished from malignant mixed müllerian tumour which occurs in the same age group and has carcinomatous and sarcomatous components. However, these tumours are generally solid, lack the characteristic squamous epithelial lined cyst and show different mucin expression. Immature malignant teratoma occurs in a younger age group and shows a variety of lines of mesenchymal differentiation, notably towards neural elements, which were absent in this case.

Germ cell tumours of all types derive from neoplastic totipotent stem cells whose progeny retain the ability to differentiate towards tissues of all three embryonic somatic layers and the transitional extraembryonal structures. The original pattern of the better differentiated members of this group suggests that local cellular influences are important in determining the final phenotype expressed by individual cells. The histological behaviour and histological appearances of these tumours are determined by the degree to which this embryogenetic environmental responsiveness is retained. In contrast, our case resembles carcinosarcomas at other sites in that there was an essentially random mixture of stromal and epithelial elements. This reflects a probabilistic commitment of individual cells and clones to one or other expressed phenotype without regard to organogenetic influences. Our case implies glandular malignant transformation within the previously benign, fully differentiated, glandular component of a mature cystic teratoma, although no premalignant changes were identified despite thorough sampling.

Adenocarcinomas are the second most common malignancies arising within dermoid cysts $^{1}$ and may display gastrointestinal phenotypes, but lack the malignant stroma as in our case. Sarcomas alone or in combination with squamous carcinoma ${ }^{56}$ have been described arising in a mature cystic teratoma. To the best of our knowledge, no case of sarcoma arising in association with adenocarcinoma has been described before.

The prognosis of patients with malignant transformation in teratoma is very poor, with most women dying within one year. ${ }^{1}$ Occasional cases with prolonged survival have been reported. $^{7}$ Poor prognostic factors include tumour dissemination, cyst wall invasion, ascites, spontaneous or accidental rupture, adhesions, and tumour type other than squamous carcinoma. Treatment is surgical, with pelvic clearance in most cases. Chemotherapy may be indicated according to the tumour type.

1 Russell P, Bannatyne P. Surgical pathology of the ovaries. Edinburgh: Churchill Livingstone, 1989:436-40.

2 Chang DH, Sueh S, Soong YK. Small cell carcinoma with neurosecretory granules arising in an ovarian dermoid cyst. Gynecol Oncol 1992;46:246-50.

3 Morimitsu Y, Nakashima O, Nakashima Y, Kojiro M, Shimokobe T. Apocrine adenocarcinoma arising in cystic teratoma of the ovary. Arch Pathol Lab Med 1993; 117:647-9.

4 Randall BJ, Ritchie C, Hutchison RS. Paget's disease and invasive undifferentiated carcinoma occurring in a mature cystic teratoma of the ovary. Histopathology 1991;18:469-

5 Genadry R, Parmley T, Woodruff JD. Secondary malignancies in benign cystic teratomas. Gynecol Oncol 1979;8:24651.

6 Hanada M, Tsujimura T, Shimizu H. Multiple malignancies (squamous cell carcinoma and sarcoma) arising in a
dermoid cyst of the ovary. Acta Pathol fpn 1981;31:681-8.

7 Ueda G, Fugita M, Ogawa H, Sawada $M$, Inoue $M$ Tanizawa $O$. Adenocarcinoma in a benign cystic teratoma of the ovary: report of a case with a long survival period. Gynecol Oncol 1993;48:259-63.

\title{
Tenascin in human papillomavirus associated lesions of the uterine cervix
}

Department of

Pathology,

University of Oulu,

Oulu, Finland

R Pöllänen

Y Soini

V-P Lehto

Department of

Obstetrics and

Gynaecology

$S$ Vuopala

Department of Public Health Sciences and General Practice

E Läärä

Correspondence to: DrY Soini,

Department of Pathology,

University of Oulu,

Kajaanintie $52 \mathrm{D}$,

FIN-90220 Oulu,

Finland.

Accepted for publication 4 December 1995

\author{
R Pöllänen, Y Soini, S Vuopala, E Läärä, V-P Lehto
}

Abstract

The immunohistochemical expression of tenascin was studied in 80 morphologically diagnosed condylomas and cervical intraepithelial neoplasia (CIN) lesions. The results were compared with the human papillomavirus (HPV) DNA subtype, which was determined by HPV dot blot and in situ hybridisation. Tenascin mRNA synthesis was also determined in 10 selected cases by in situ hybridisation. No statistically significant association was found between tenascin expression and the degree of dysplasia or the HPV subtype. There was, however, a strong cor- relation between the extent of tenascin immunoreactivity and the degree of inflammation. Synthesis of tenascin mRNA was detected in basal keratinocytes and in fibroblasts by in situ hybridisation. The lack of association between the grade of CIN and tenascin expression precludes its use as a marker of premalignancy in CIN. (f Clin Pathol 1996;49:521-523)

Keywords: tenascin, extracellular matrix proteins, human papillomavirus infection, HPV DNA, inflammation.

Human papillomavirus (HPV) is the aetiological agent of various genital lesions and there is 
Table 1 Distribution of HPV positive and negative patients and tenascin immunoreactivity in the cervical lesions

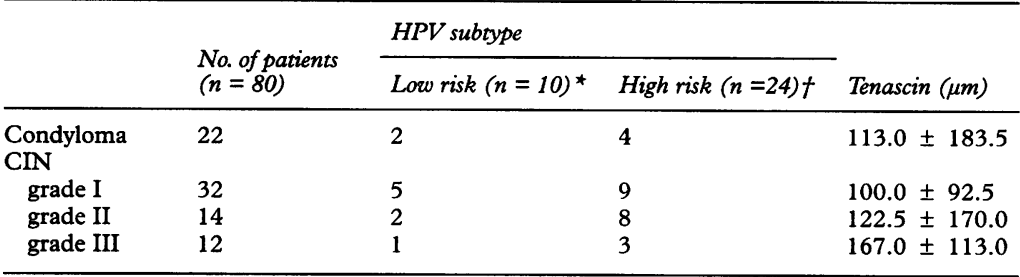

${ }^{\star}$ HPV 6 or $11 ;$ HHPV $16,18,31,33$, or 35 . Tenascin immunoreactivity was scored as the maximal width of the anti-tenascin reactive zone, measured using an ocular micrometer.

a significant correlation between infection with specific HPV subtypes and the nature of the lesion. ${ }^{1}$ Thus, HPV types 6 and 11 are most commonly found in association with benign condylomata whereas HPV types 16,18 and 31 are found in high grade dysplasia and cervical cancer. ${ }^{2}$

Tenascin is an extracellular matrix protein that plays a role in tissue interactions during fetal development and in malignant transformation. ${ }^{4-6}$ It is involved in critical functions such as epithelial-mesenchymal interactions, organogenesis, somatic growth regulation, epithelial renewal, cell adhesion, morphogenesis, and cell migration. ${ }^{4-6}$

To determine whether tenascin expression is correlated with the degree of cervical dysplasia and the HPV subtype, we investigated its expression in 80 condylomas and cervical intraepithelial neoplasia (CIN) lesions and correlated the results with the HPV subtypes present in these lesions.

\section{Methods}

Samples for the study were obtained at colposcopy from 80 patients. Nine patients with normal histology or with cervicitis with no indication of condyloma or dysplasia were also included. Biopsy specimens for routine histological evaluation were fixed in $10 \%$ formalin, processed routinely, and embedded in paraffin wax using standard techniques. Histological diagnosis was made using generally accepted criteria. ${ }^{7}$ The extent of the inflammatory reaction was scored as follows: none $(-)$; weak $(+)$; moderate $(++)$; and strong $(+++)$.

HPV DNA was detected by dot blot hybridisation using the Virapap HPV DNA screening kit, the Viratype HPV DNA typing kit (Digene, Gaithersburg, Maryland, USA) and the Affiprobe kit (Orion, Helsinki, Finland) according to the manufacturers' instructions. HPV DNA was detected by the Virapap HPV DNA screening kit, using a mixture of ${ }^{32} \mathrm{P}$-labelled RNA probes detecting HPV types $6,11,16$, $18,31,33$, and 35 . Specimens displaying a positive hybridisation signal were typed by using the Viratype kit or the Affiprobe kit with probes delineating three $(6 / 11,16 / 18$ and $31 / 33 / 35)$ or four viral groups $(6 / 11,16,18 / 33$, and 31 ), respectively.

In situ DNA hybridisation was used in cases with negative scores on dot blot hybridisation. For that purpose, the Biohit in situ HPV DNA typing test kit (Biohit, Helsinki, Finland), containing biotin labelled probes for HPV types 6 , $11,16,18,31,33$, and 35 , was used as described by the manufacturer. Positive controls included cervical samples positive for each of the HPV subtypes tested, and HPV 16 containing CaSki cells provided with the kit.

For immunohistochemistry, the sections were incubated with a monoclonal tenascin mouse antibody (143CB7), ${ }^{8}$ and then with the biotinylated rabbit anti-mouse antibody and the avidinbiotin peroxidase complex (Dako, Glostrup, Denmark). Diaminobenzidine was used as the chromogen. Negative control consisted of substituting the primary antibody with posphate buffered saline and normal mouse serum. The maximal width of the anti-tenascin reactive subepithelial zone was measured by using an ocular micrometer and the results were divided semiquantitatively into four groups as follows: $+=$ $1-25 \mu \mathrm{m} ;++=26-100 \mu \mathrm{m} ;+++=101-250 \mu \mathrm{m}$; $++++=>250 \mu \mathrm{m}$ maximal distance from the basal epithelium.

A cDNA fragment (bases 814-1316) of the full length tenascin $\mathrm{cDNA}^{9}$ was synthesised by PCR from the HT-11 subclone, kindly provided by Dr Luciano Zardi (Instituto Nazionale Per La Ricerca Sul Cancro, Genova, Italy), by using the following primers: 5' CCCTGCAGTGAGGAGCACGGCACA $3^{\prime}$ and $5^{\prime}$ TGCCCATTGACACAGCGGCCATGG 3'.

The cDNA (503 base pairs) obtained by PCR was subcloned into a TA vector (TA cloning Kit, Invitrogen, USA). Sense and antisense RNA probes were generated from a linearised template by using a riboprobe transcription kit (Promega, Maddison, Wisconsin, USA) and the probes were labelled with ${ }^{35}$ S-UTP (Amersham, Little Chalfont, UK). Hybridisation, post-hybridisation washes and detection of the hybrids were carried out as described previously. ${ }^{10}$

\section{Results}

In all 80 cases HPV infection was diagnosed on the basis of characteristic morphological features. HPV DNA was detected by in situ or dot blot hybridisation in $32(40 \%)$ patients. The low risk HPV subtypes ( 6 or 11 ) were detected in 10 patients and the high risk subtypes (16, $18,31,33$, and 35 ) in 24 (table 1).

The extent of the tenascin positive zone in relation to the morphology of the lesion, the presence or absence of HPV DNA and the degree of inflammation is shown in tables 1 and 2. There was no statistically significant difference in the extent of tenascin immunoreactivity between the different CIN lesions. Patients with no or weak inflammation showed significantly less tenascin immunoreactivity $(91.5 \pm 114.0$ $\mu \mathrm{m})$ than those with strong inflammation (185.5 $\pm 175.5 \mu \mathrm{m})$ ( $\mathrm{p}=0.002$, Fisher's exact test). There was no significant difference in tenascin immunoreactivity between patients with benign $(71.0 \pm 93.0 \mu \mathrm{m})$ and malignant $(88.5 \pm 84.5 \mu \mathrm{m})$ HPV subtypes ( $p=0.84$, Fisher's exact test). According to a three-way analysis of variance, inflammation had a statistically significant $(p=$ 0.03 ) effect on the accumulation of tenascin but not on the degree of dysplasia $(p=0.25)$ or the HPV subtype $(p=0.44)$.

In the 10 cases studied by in situ hybridisation for tenascin mRNA, labelling was ob- 
Table 2 Extent of the tenascin positive zone in relation to the degree of dysplasia, the presence of HPV and the degree of inflammation

\begin{tabular}{lrrrl}
\hline & \multicolumn{5}{c}{ Tenascin } & immunoreactivity $(n ; \mu m)^{*}$ \\
\cline { 2 - 5 } & $1-25$ & $26-100$ & $101-250$ & $>250$ \\
\hline Dysplasia & & & & \\
CIN 0 & 11 & 5 & 3 & 3 \\
CIN I & 12 & 8 & 11 & 1 \\
CIN II & 5 & 4 & 4 & 1 \\
CIN III & 1 & 5 & 4 & 2 \\
HPV subtype & & 11 & 14 & 6 \\
not detected & 15 & 11 & 3 & 0 \\
low risk & 6 & 1 & 4 & 1 \\
high risk & 7 & 12 & 5 & 1 \\
Inflammation & & & 7 & 2 \\
none & 16 & 8 & 7 & 3 \\
weak & 8 & 11 & 3 & 1 \\
moderate & 4 & 2 & 2 & 5 \\
strong & 0 & 2 & 5 & \\
\hline
\end{tabular}

$\star_{+}=1-25 \mu \mathrm{m} ;++=26-100 \mu \mathrm{m} ;+++=101-250 \mu \mathrm{m}$ $++++=>250 \mu \mathrm{m}$ maximal distance from the basal epithelium; $\mathrm{n}=$ number of patients.

Associations by Fisher's exact test: CIN $0-\mathrm{I} / \mathrm{CIN}$ II-III versus low $(+,++) /$ high $(+++,++++)$ tenascin immunoreactivity, $\mathrm{p}=0.22$

HPV benign/HPV malignant versus low $(+,++) /$ high $(+++,++++)$ tenascin immunoreactivity, $\mathrm{p}=0.84$.

Inflammation weak $(-,+)$ /strong $(++,+++)$ versus low $(+,++) /$ high $(+++,++++)$ tenascin immunoreactivity $\mathrm{p}=0.002$.

served in basal keratinocytes and subepithelial fibroblasts, especially at sites of inflammation. The labelling intensity of the basal keratinocytes and fibroblasts correlated with tenascin expression in the subepithelial connective tissues. No labelling was seen in normal cervical samples lacking inflammation.

\section{Discussion}

In this study tenascin immunoreactivity was investigated in 80 cervical condylomas and $\mathrm{CIN}$ lesions. While no association was found between tenascin immunoreactivity and the degree of cervical dysplasia, statistical analysis showed that enhanced tenascin expression in patients with $\mathrm{CIN}$ was due to cervicitis, which frequently accompanies CIN. This suggests that inflammatory cells are responsible for the induction of tenascin synthesis in cervicitis, probably by releasing cytokines and growth factors, such as transforming growth factor $\beta$, which has been shown to stimulate fibroblasts to synthesise tenascin. ${ }^{11}$ Indeed, in in situ experiments, we observed signals for tenascin mRNA in fibroblasts present in the inflammatory infiltrate. Strong signals were also detected in basal keratinocytes, suggesting that these cells also participate in tenascin synthesis.

Tiitta et $a l^{8}$ observed enhanced tenascin expression in cervical and vulvar lesions containing HPV DNA. However, in their study, only in a subset of samples were probed for HPV DNA and no definite conclusions could be drawn as to whether it is the degree of dysplasia, HPV subtype or some other factor that was responsible for the enhanced expression of tenascin. In the present study in which in situ hybridisation and dot blot hybridisation were carried out on a large number of cases, we showed conclusively that tenascin accumulation is mainly due to the inflammatory component of these lesions. Consequently, tenascin cannot be used as a marker of the biological potential of dysplastic cervical lesions.

Tenascin cDNA was kindly provided by Dr Luciano Zardi, Instituto Nazionale Per La Ricerca Sul Cancro, Genova, Italy.

We thank Professor Ismo Virtanen, Department of Anatomy, University of Helsinki, Helsinki, Finland, for providing the antitenascin antibody. The skillful technical assistance of Ms Mirja Vahera and $\mathrm{Mr}$ Tapio Leinonen is kindly acknowledged. This study was supported by grants from the Academy of Finland, the Finnish Cancer Society, the Cancer Society of Northern the Finnish Cancer Socius Finland, the Sigrid Juselius Foundation, the Finnish Cancer Research Fund, the Finnish Anti-Tuberculosis Association, the Ida Montin Foundation, and the Finnish Virologist's Founda-
tion. RP is a recipient of a University of Oulu Post Graduate tion. RP

1 Koss LG. Carcinogenesis in the uterine cervix and human papillomavirus infection. In: Syrjänen K, Gissman L, Koss LG, eds. Papillomavirus and human disease. Berlin: Springer Verlag, 1987:235-67.

2 Lorincz AT, Temple GF, Kurman RJ, Jenson AB, Lancaster WD. Oncogenic association of specific human papillomavirus types with cervical neoplasia. $\mathcal{F}$ Natl Cancer Inst 1987;79:671-7.

3 Pfister H. Human papillomaviruses and genital cancer. $A d v$ Cancer Res 1987;48:113-47.

4 Chiquet-Ehrismann R, Mackie EJ, Pearson CA, Sakakura T. An extracellular matrix protein involved in tissue interactions during fetal development and oncogenesis. Cell $1986 ; 47: 131-2$.

5 Inaguma Y, Kusakabe M, Mackie EJ, Pearson CA, ChiquetEhrismann R, Sakakura T. Epithelial induction of stromal tenascin in the mouse mammary gland: From embryogenesis to carcinogenesis. Dev Biol 1988;128:245-55.

6 Edelman GM, Jones FS. Cytotactin: a morphoregulatory molecule and a target for regulation by homeobox gene molecule and a target for regulation by hom
products. Trends Biochem Sci 1992;1 7:228-32.

7 Ferenczy A, Winkler B. Cervical intraepithelial neoplasia and condylomata. In: Kurman RJ, ed. Blaustein's pathology of the female genital tract. 3rd edn. New York: Springer, 1987:177-217.

8 Tiitta O, Wahlström T, Paavonen J, Linnala A, Sharma S, Gould VE, Virtanen I. Enhanced tenascin expression in cervicar vulvar koilocytic lesions. Am f Pathol 1992; 141:907-13.

9 Siri A, Carnemolla B, Saginati M, Leprini A, Casari G, Baralle $F$, Zardi $L$. Human tenascin: primary structure, pre-mRNA splicing patterns and localization of the epitopes recognized by two monoclonal antibodies. Nucleic Acids Res 1991;19:525-31.

10 Autio-Harmainen H, Hurskainen T, Niskasaari K, Höyhtyä $\mathrm{M}$, Tryggvason $\mathrm{K}$. Simultaneous expression of 70 kilodalton Type IV collagenase and Type IV collagen alpha-1(IV) chain genes by cells of early placenta and gestational chain genes by cells of early placenta and

11 Pearson CA, Pearson D, Shibahara S, Hofsteenge J, Chiquet-Ehrismann R. Tenascin: cDNA cloning and induction by TGF- $\beta$. EMBO $f$ 1988;7:2877-982. 